\title{
Dual-band Dielectric Resonant Antenna
}

\author{
Tsung-Shan Yung, Tze-Hsuan Chang*, Wen-Zhou Wu, and Jean-Fu Kiang \\ Department of Electrical Engineering and \\ Graduate Institute of Communication Engineering \\ National Taiwan University, Taipei, Taiwan, ROC \\ E-mail: jfkiang@cc.ee.ntu.edu.tw
}

\begin{abstract}
In this paper, dielectric resonant (DR) antennas with inserted slit or well are proposed and verified by HFSS simulation. It is found that broadband and dual-band operation can be achieved by the perturbation of air gap.
\end{abstract}

\section{Introduction}

DR made of low-loss and high-permittivity material has been widely used to implement microwave components. DR antennas are suitable for millimeterwave due to their low loss. Unlike patch antennas, DR antennas can radiate from all surfaces, rendering high radiation efficiency and low $Q$-factor [1].

Each resonant mode in DR has different internal field distribution and hence different radiation characteristics. Moreover, mode degeneracy and hybrid modes always exist in spherical DR and cylindrical DR, which increase cross-polarization levels and deteriorate their performance. Resonant frequency of rectangular DR can be uniquely determined by the dimensions of the resonator without mode degeneracy problem. However, rectangular DR antennas are not widely used partially because their resonant modes have not been well categorized [2].

Stacking two DRs of different size increases the operating bandwidth [3]. A conical DR antenna having wide bandwidth is viewed as stacking several resonators with gradual radius change [4]. Placing parasitic DRs on both sides of a driving DR can also increase the bandwidth or obtain dual bands.

\section{Concept and Mechanism}

Antennas with lower $Q$-factor have boarder bandwidth. Thus, the technology of reducing $Q$-factor has been applied on DR antenna design to increase its bandwidth. Ring structures, which occupy less volume and result in lower $Q$-factor, are utilized to enhance its bandwidth and obtain higher resonant frequency [5]. It is found that a center-fed cylindrical DR antenna with air gap between the DR and ground plane has a wider bandwidth as the thickness of the gap is increased.

By image theory, the half-split DR with an air gap can be transformed into 
two half-split DRs separated by an air gap. Thin air gap between the two half-split DRs creates discontinuity of normal electric field at the interface between dielectric and air, and the electric field normal to the interface is much stronger in the air gap than inside the DR. The thin air gap also has significant effect upon the input impedance and resonant frequency. Increasing gap thickness broadens the bandwidth, increases the antenna resonant frequencies, decreases the maximum value of the input resistance, reduces $Q$-factor, and increases radiation efficiency. However, it is difficult to fabricate. Thus, an air gap inserted into DR antenna is proposed to achieve wider bandwidth and keep the same size as an intact DR antenna.

\section{Results and Conclusions}

As shown in Figure 1, a narrow slot is drilled in the middle of a rectangular DR antenna, where the strongest electric field of the $\mathrm{TE}_{111}^{z}$ mode appears normal to the slit wall. Contrast of permittivity between air and dielectric further enhances the electric field in the slit. However, the slit only slightly affects the electric field of the $\mathrm{TE}_{211}^{z}$ mode because the slit is parallel to the electric field of that mode. Thus, impedance variation of the $T E_{111}^{z}$ mode is broadened more significantly with slit width increase than the $T E_{211}^{z}$ mode. The resonant frequencies of both the $T E_{111}^{z}$ and $T E_{211}^{z}$ modes are increased with increasing slit width due to perturbation, as shown in Figure 2(a). When slit is placed asymmetrically with respected to the center axis, where the strongest electric field associated with $\mathrm{TE}_{211}^{z}$ mode is normal to the slit wall. Hence, the side slit is expected to perturb the $\mathrm{TE}_{211}^{z}$ mode as well as the $\mathrm{TE}_{11}^{z}$ mode, as shown in Figure 2(b).

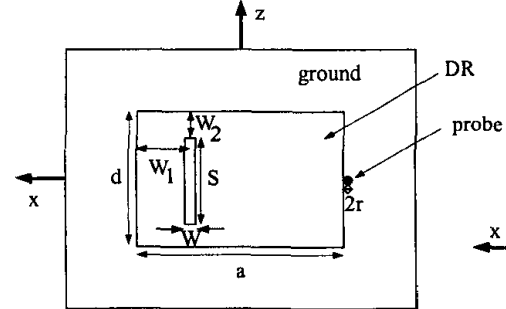

(a)

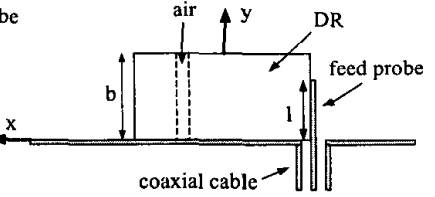

(b)

Figure 1 Rectangular DR antenna with a side slit, (a) top view, (b) side view. 


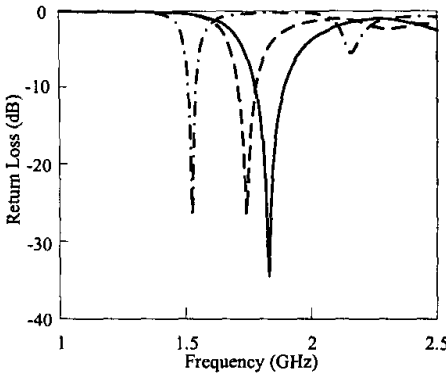

(a)

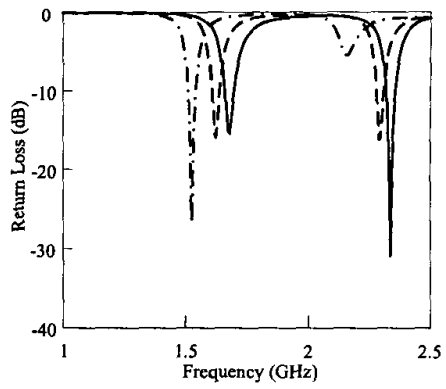

(b)

Figure 2 Return loss versus frequency for the rectangular DR antenna with different slit widths, $\varepsilon_{\mathrm{r}}=40, a=26.5 \mathrm{~mm}, b=13 \mathrm{~mm}, d=19.4 \mathrm{~mm}, 2 r=1 \mathrm{~mm}, 1=8.5 \mathrm{~mm}, S=13.4 \mathrm{~mm}, \cdots-\cdot$ No slit, -.-: $W=2 \mathrm{~mm},-: W=4 \mathrm{~mm}$, (a) center slit, $W_{1}=a / 2, W_{2}=(d-S) / 2$, (b) side slit, $W_{1}=5.75 \mathrm{~mm}, W_{2}=3 \mathrm{~mm}$.

The side slit also eliminates the null along the $\hat{y}$ direction to have similar radiation pattern to the $\mathrm{TE}_{111}^{2}$ mode. This feature can be utilized to design a dual-band DR antenna. Figure 3 shows a rectangular DR antenna with a rectangular well which is used to improve impedance matching at both the $\mathrm{TE}_{111}^{z}$ and $\mathrm{TE}_{211}^{z}$ modes. The well is much wider than a slit, and placed asymmetrically with respect to the $y$ axis. Figure 4 (a) shows good return loss performance in the $1.8 \mathrm{GHz}$ and $2.4 \mathrm{GHz}$ bands with bandwidth of $4.8 \%$ and $2.26 \%$, respectively. The bandwidth can be further increased by using lower-permittivity dielectric, as shown in Figure 4 (b).

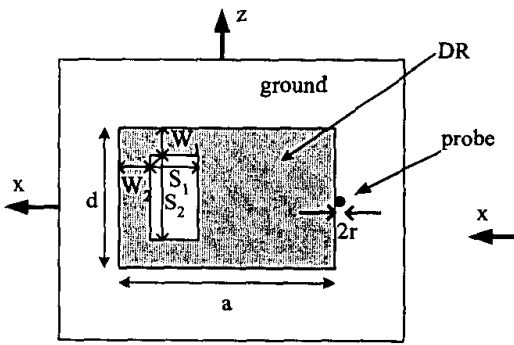

(a)

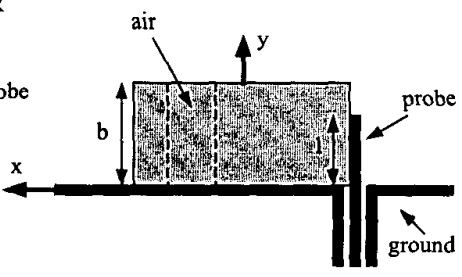

(b)

Figure 3 Rectangular DR antenna with a well, (a) top view, (b) side view. 


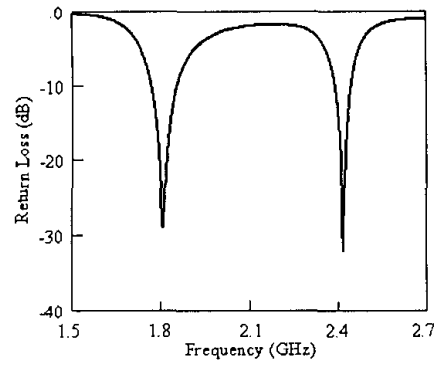

(a)

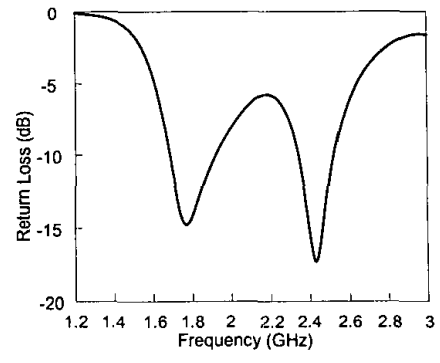

(b)

Figure 4 Return loss of dual-band DR antenna, $a=40 \mathrm{~mm}, b=16$ am, $d=21 \mathrm{~mm}, 2 r=1 \mathrm{mLl}, 1=16$ min, $W_{1}=W_{2}=4 \mathrm{~mm}, S_{1}=10 \mathrm{~mm}, S_{2}=13 \mathrm{mlml}$, (a) $\varepsilon_{\mathrm{r}}=40$, (b) $\varepsilon_{\mathrm{r}}=20$.

Acknowledgment: This work is sponsored by the National Science Council, ROC, under contract NSC 92-2213-E-002-070.

\section{Reference}

[1] D. Kajfez and P. Guillon, Eds., Dielectric Resonator. Norwood. MA: Artech House, 1986.

[2] R. K. Mongia and P. Bhartia, "Dielectric resonator antennas-A review and general design relations for resonant frequency and bandwidth," Int. J. Microwave Millimeter Wave Comput.-Aided Eng., vol.4, no.3, pp.230-247, 1994.

[3] S. M. Shum and K. M. Luk, "Stacked annular ring dielectric resonator antenna excited by axis-symmetric coaxial probe," IEEE Trans. Antennas Propagat., vol.43, no.8, pp.889-892, Aug. 1995.

[4] A. A. Kishk, Y. Tin, and A. W. Glisson, "Conical dielectric resonator antennas for wideband applications," IEEE Trans. Antennas Propagat., vol.50, no.4, pp.469-474, Apr. 2002.

[5] M. Verplanken and J. Van Bladel, "The electric-dipole resonances of ring resonators of very high permittivity," IEEE Trans. Microwave Theory Tech., pp.108-112, Feb. 1976. 